\title{
Abnormal primary and permanent dentitions with ectodermal symptoms predict WNT10A deficiency
}

\author{
Birgitta Bergendal ${ }^{1,2^{*}} \mathbb{D}$, Johanna Norderyd ${ }^{1,2}$, Xiaolei Zhou ${ }^{3,4}$, Joakim $\mathrm{Klar}^{3,4}$ and Niklas Dahl ${ }^{3,4}$
}

\begin{abstract}
Background: The WNT10A protein is critical for the development of ectodermal appendages. Variants in the WNT10A gene may be associated with a spectrum of ectodermal abnormalities including extensive tooth agenesis.

Methods: In seven patients with severe tooth agenesis we identified anomalies in primary dentition and additional ectodermal symptoms, and assessed WNT10A mutations by genetic analysis.

Results: Investigation of primary dentition revealed peg-shaped crowns of primary mandibular incisors and three individuals had agenesis of at least two primary teeth. The permanent dentition was severely affected in all individuals with a mean of 21 missing teeth. Primary teeth were most often present in positions were succedaneous teeth were missing. Furthermore, most existing molars had taurodontism. Light, brittle or coarse hair was reported in all seven individuals, hyperhidrosis of palms and soles in six individuals and nail anomalies in two individuals. The anomalies in primary dentition preceded most of the additional ectodermal symptoms. Genetic analysis revealed that all seven individuals were homozygous or compound heterozygous for WNT10A mutations resulting in C107X, E222X and F228l.

Conclusions: We conclude that tooth agenesis and/or peg-shaped crowns of primary mandibular incisors, severe oligodontia of permanent dentition as well as ectodermal symptoms of varying severity may be predictors of biallelic WNT10A mutations of importance for diagnosis, counselling and follow-up.
\end{abstract}

Keywords: WNT10A mutations, Ectodermal dysplasia, Dental, Primary dentition, Permanent dentition

\section{Background}

The WNT10A (Wingless-type MMTV integration site family, member 10A) gene is a member of the WNT gene family that has been implicated in oncogenesis and in several developmental processes including tooth formation [1, 2]. Bi-allelic WNT10A mutations cause a spectrum of symptoms from ectodermal appendages. Patients may present with nail dystrophy, palmoplantar hyperkeratosis, hyperhidrosis as well as hypohidrosis, sparse or brittle hair, eye-lid cysts and keratoconus in different combinations and severity in a continuum of phenotypes [3-6]. In some cases the constellation of features may be defined as a specific clinical entity

\footnotetext{
* Correspondence: birgitta.bergendal@ril.se

${ }^{1}$ National Oral Disability Centre for Rare Disorders, The Institute for Postgraduate Dental Education, P.O. Box 1030SE- 55111 Jönköping, Sweden ${ }^{2}$ School of Health and Welfare, Jönköping University, Jönköping, Sweden Full list of author information is available at the end of the article
}

among the ectodermal dysplasias (EDs), e.g., odontoonycho-dermal dysplasia (OODD; OMIM 257980) or Schöpf-Schultz-Passarge syndrome (SSPS; OMIM 224750) [3-5, 7]. The most consistent and specific symptom in cases with either bi-allelic or mono-allelic WNT10A mutations reported to date is tooth agenesis of the permanent dentition [3, 4, 6, 8-14]. Studies of patients with non-syndromic oligodontia (agenesis of 6-28 teeth) referred for centralized treatments revealed a carrier frequency of up to $56 \%$ for WNT10A mutations [8]. However, a population-based study of Swedish individuals with isolated oligodontia revealed that the frequency of at least one WNT10A mutation in non-related probands was 28\% [11]. When adding mutations in the genes MSX1, PAX9, AXIN2, EDA, EDAR, and EDAR$A D D$ in the same material the frequency of genetically verified isolated oligodontia reached $38.3 \%$. 
Taken together, independent reports from us and others indicate that WNT10A mutations are the most frequent specific cause of isolated hypodontia (agenesis of at least one tooth), and oligodontia (agenesis of six or more teeth, third molars excluded) of the permanent dentition known to date. The different frequencies in WNT10A associated tooth anomalies in these studies may be related to ethnicity, phenotypic differences and selection criteria of study participants. Noteworthy, mutations in the WNT co-receptor LRP6 gene were recently identified in rare cases of isolated oligodontia bringing further evidence for the importance of WNT10A in tooth development [15].

Bi-allelic WNT10A mutations are usually associated with a larger number of missing teeth when compared to mono-allelic mutations [3, 4, 6, 8-11, 14]. Noteworthy, the penetrance among heterozygotes is incomplete and a considerable proportion of carriers do not express any tooth anomalies at all [12]. In addition to tooth agenesis, WNT10A variants may cause abnormal shape of both roots and crowns of permanent teeth $[16,17]$. Deformities include taurodontism of molars, misshaped crowns and peg-shaped incisors [10, 14, 16, 17]. Taurodontism is a condition in human molars in which the body of the tooth is enlarged vertically at the expense of the roots, causing the pulp chamber to extend apically below the cementoenamel junction $[18,19]$. Thus, isolated hypoor oligodontia, and tapered frontal permanent teeth give reasons to suspect underlying WNT10A mutations. Also, a less disturbed primary dentition with conical frontal teeth was reported by Bohring et al., 2009 [4].

We hypothesized that the combined assessment of primary and permanent dentition as well as search for mild or unnoticed ectodermal symptoms may identify carriers of WNT10A mutations. In this study we present seven individuals with severe tooth anomalies in whom symptoms from other ectodermal appendages were identified. We made a detailed characterization of both the primary and permanent dentition together with symptoms from other ectodermal derivatives. Our aim was to evaluate if abnormalities in both primary and permanent dentitions, together with other ectodermal symptoms, may serve as predictors for WNT10A deficiency of diagnostic importance early in life.

\section{Methods}

\section{Clinical investigations}

Seven individuals with oligodontia and other ectodermal symptoms diagnosed at the National Oral Disability Centre for Rare Disorders in Jönköping, Sweden, were retrospectively selected when we identified typical abnormalities in their primary dentition. Two of the patients (Ia and $\mathrm{Ib}$ ) were siblings and the remaining were probands from five families. Three patients were females and four were males. Clinical data were compiled from patient records including photographs and panoramic radiographs. Evaluation of taurodontism of primary or permanent mandibular molars was performed using the method described by Seow and Lai, 1989 [20], using panoramic radiographs to calculate the quotient of crown-body $(\mathrm{CB})$ and root $(\mathrm{R})$ length. We used the threshold value for taurodontism of 0.9 or more as suggested by Kan et al., 2010 [21]. The measure points for $\mathrm{CB}$ and $\mathrm{R}$ were fixed by two of the authors separately from the molar segments of the mandible on panoramic radiographs. The accuracy of the measuring points were evaluated simultaneously and adjusted by two specialists in dentomaxillofacial radiology before calculation of the $\mathrm{CB}: \mathrm{R}$ ratio to establish taurodontism. Ectodermal signs and symptoms were compiled from anamnestic data in patient records and by telephone interviews in order to obtain specific information.

\section{Genetic analysis}

The patients and their parents were sampled and tested for mutations in the WNT10A gene. All four exons of WNT10A, exon-intron boundaries and $5^{\prime}$ and $3^{\prime}$-UTRs were PCR amplified using primers designed via the Primer3 software (sequences available upon request) and checked with ePCR using the UCSC genome browser. Mutation screening was performed of all coding parts of the WNT10A gene by bi-directional Sanger sequencing using Big Dye Terminator v3.1 cycle sequencing kit (Applied Biosystems, Foster City, CA) and separated on an ABI 3730xl DNA analyzer (Applied Biosystems; primers available upon request). Chromatograms were aligned and compared to the reference sequence of the WNT10A gene (NM_025216.2).

\section{Results \\ Dental characteristics}

Three out of the seven individuals (Ia, II and III) had agenesis of primary teeth (range 2-3) including five maxillary and two mandibular lateral incisors, $5 \%$ of all primary teeth in the cohort. In four individuals the mandibular primary incisors were present with peg-shaped crowns. In two individuals (II and V) the primary incisors were severely worn and there was no evidence of the original crown shape, and in one (IV) the primary incisors were lost at time of examination. Thus, hypodontia and/or peg-shaped crowns of primary mandibular incisors were identified in 4 out of 7 patients. Furthermore, existing primary maxillary incisors in patients Ia and III were tapered, though less marked than in the mandible. When comparing genders we observed that the number of missing primary 
teeth was significantly higher among females (females $2.3 \pm 0.6, n=3$; males $=0 \pm 0, n=4 ; p=0.020$ ).

Clinical documentation including intra- and extraoral photographs, dentograms with both primary and permanent dentitions, and WNT10A mutations are shown in Fig. 1. Individual Ia had no permanent teeth at all, and individual II had only two permanent maxillary central incisors. Examination of the permanent dentition revealed extensive oligodontia with agenesis of $76 \%$ of permanent teeth (Fig. 2.). The mean number of missing permanent teeth in all seven individuals was 21.3 (range 15-28) with a distribution of $47 \%$ in the maxilla and $53 \%$ in the mandible. In the six individuals with some permanent teeth, five had severely tapered maxillary
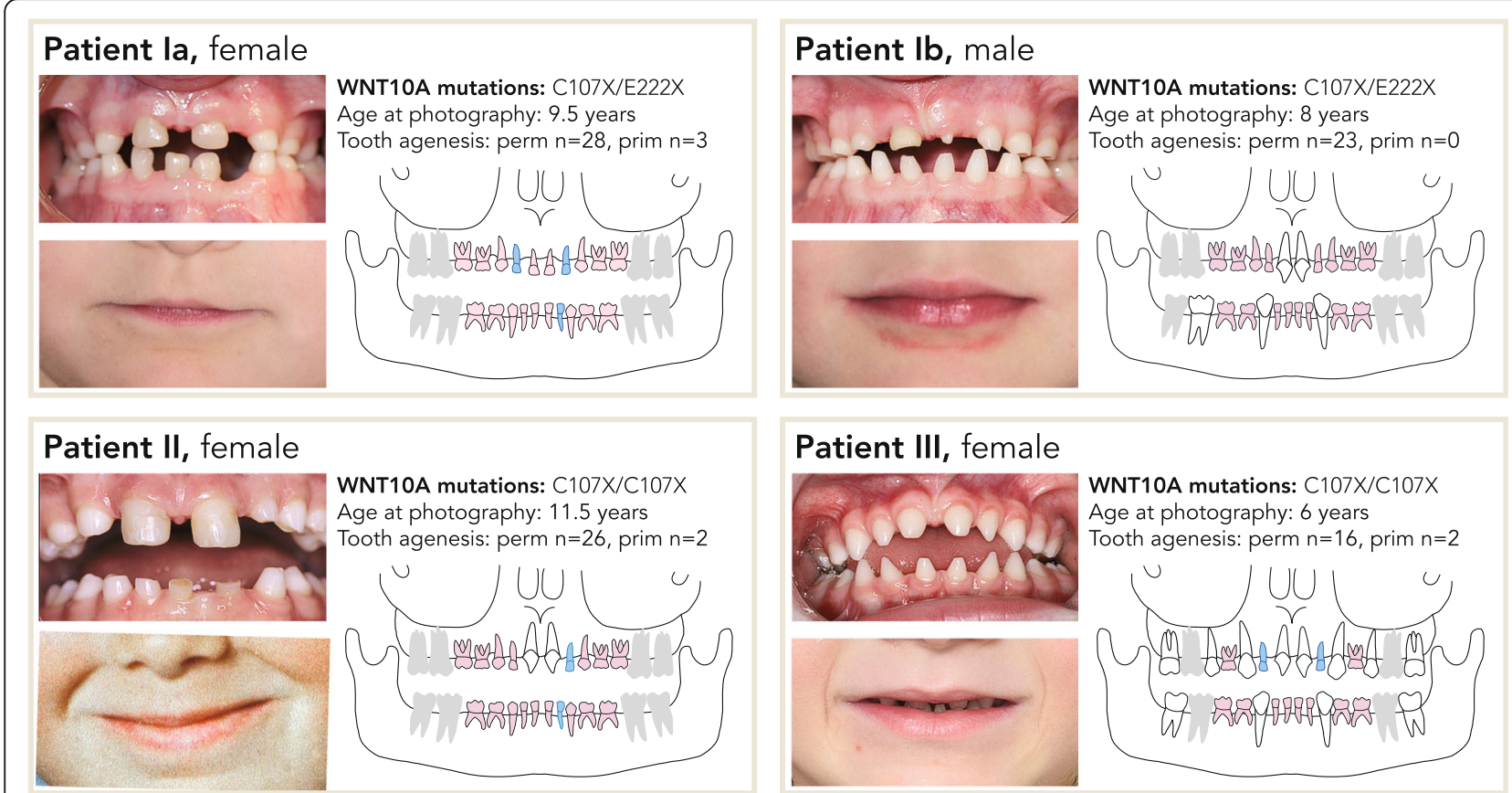

WNT10A mutations: C107X/C107X

Age at photography: 11.5 years

Tooth agenesis: perm $n=26$, prim $n=2$
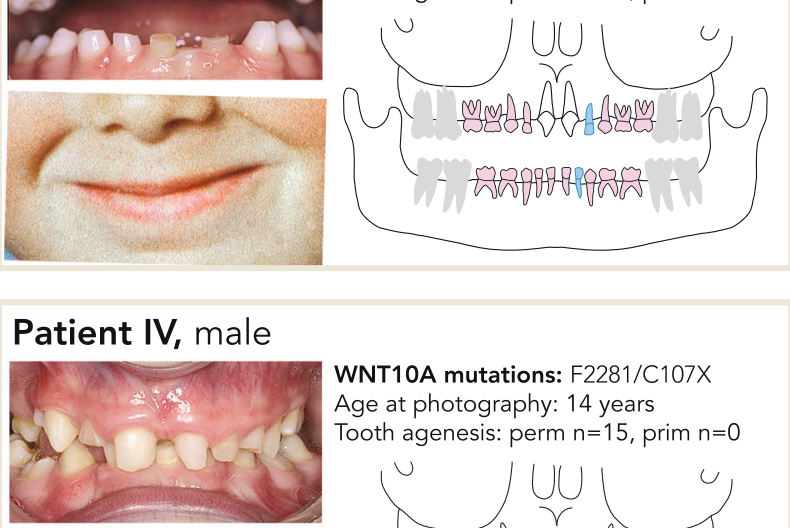

WNT10A mutations: F2281/C107X

Age at photography: 14 years

Tooth agenesis: perm $n=15$, prim $n=0$

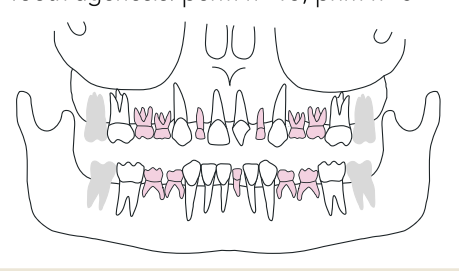

\section{Patient III, female}

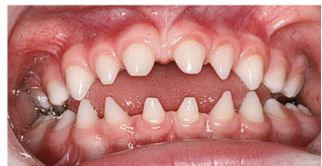

WNT10A mutations: C107X/C107X

Age at photography: 6 years

Tooth agenesis: perm $n=16$, prim $n=2$
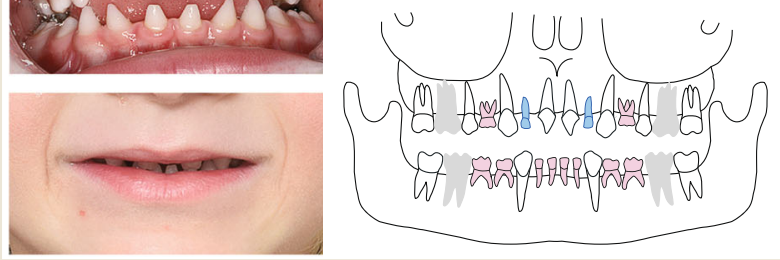

Patient V, male

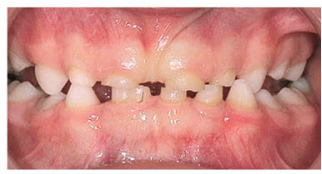

WNT10A mutations: F2281/C107X

Age at photography: 6.5 years

Tooth agenesis: perm $n=19$, prim $n=0$
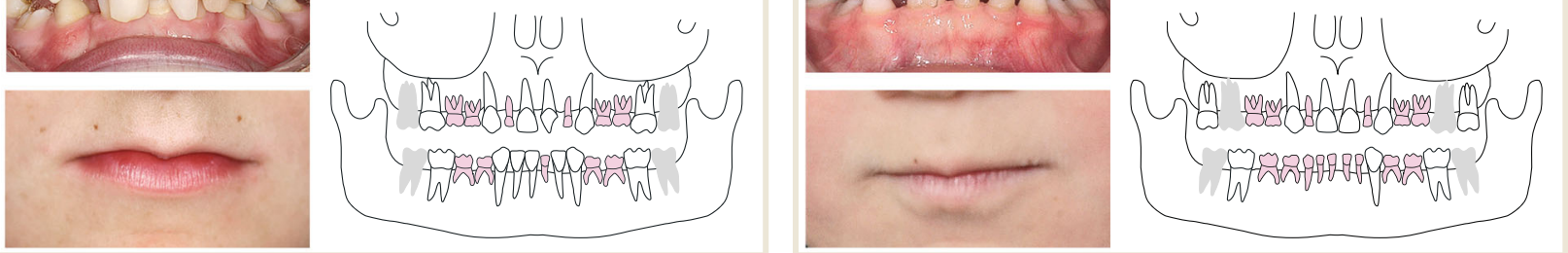

\section{Patient VI, male}

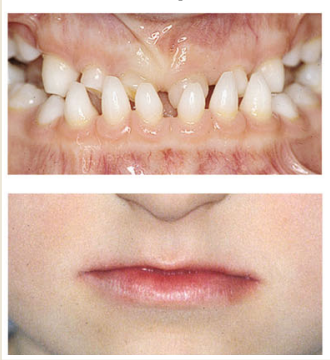

WNT10A mutations: E222X/E222X

Age at photography: 7 years

Tooth agenesis: perm $n=22$, prim $n=0$

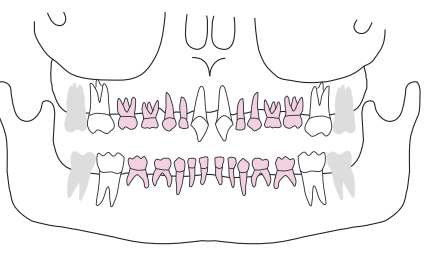

\section{Symbols in dentograms}

A Absent primary and permanent tooth

A Primary tooth without permanent successor

Absent permanent tooth

A Tapered permanent maxillary central incisor

A Permanent tooth

Fig. 1 Oral photographs, dentograms and mutations in seven individuals with bi-allelic WNT1OA mutations. Intraoral photographs show peg-shaped crowns of the primary mandibular incisors (individuals $\mathrm{la}, \mathrm{Ib}, \mathrm{III}$, and IV) and tapered maxillary primary incisors (individuals la and III). Extraoral photographs illustrate a characteristic thin upper lip seen in all but individual Ib. Individuals la and Ib are siblings 


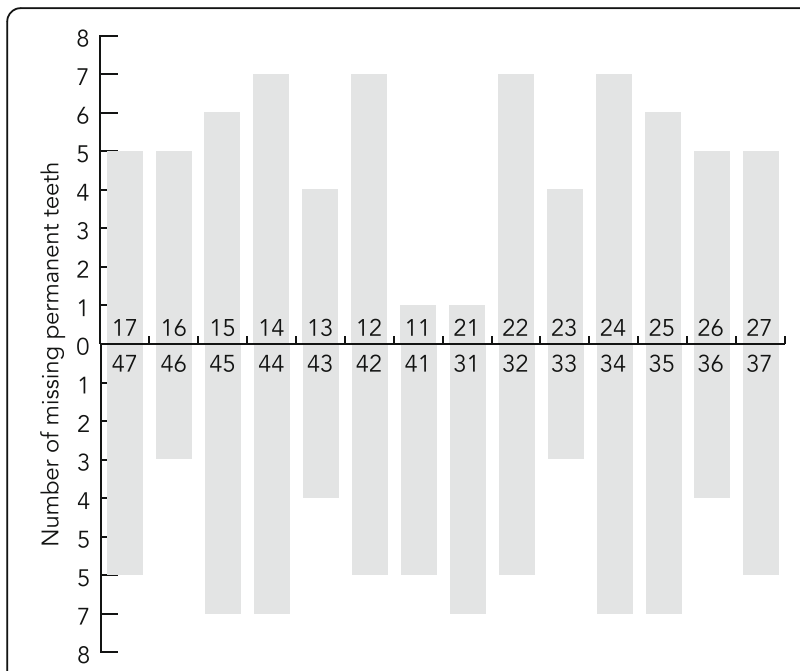

Fig. 2 Missing permanent teeth in seven individuals with bi-allelic WNT10A mutations $(n=149$, mean $=21.3$, median $=22$, range $=15-28$ ). The number of missing teeth was similar in maxilla and mandible

central incisors, nine out of 12 teeth (75\%). All seven individuals were missing maxillary lateral incisors, first premolars of both jaws, and mandibular second molars and left central incisors. The most stable teeth, present in all but in individual Ia, were the maxillary central incisors, followed by the canines in both jaws. There was no difference in number of missing permanent teeth when comparing males to females.

Taurodontism was investigated in mandibular molars using panoramic radiographs. In total, 11 primary second molars were possible to examine in six individuals; in individual IV the primary molars were partly resorbed. Nine primary molars, $82 \%$, had a score of 0.9 or more. Consequently, six individuals had taurodontism in one or both primary molars. Taurodontism of permanent dentition was confirmed in individual IV where the first molars were fully erupted. He was the only individual where evident taurodontism could be determined by cursory examination. Clinical data are summarized in Table 1.

\section{Additional ectodermal abnormalities}

Ectodermal symptoms other than tooth agenesis were in some cases unnoticed, neglected or considered normal variants by the patient/parents. Upon interview, six individuals reported to have dry skin, and the number of affected ectodermal appendages in each patient varied from 2 to 4 (mean 2.9, median 3) (Table 1). Hyperhidrosis of palms/soles was reported from five patients, with most severe symptoms in individual Ia who also showed palmo-plantar hyperkeratosis. All seven patients reported on hair abnormalities including coarse, brittle and scarce hair. Slow-growing hair was reported in individuals Ia and II with agenesis of all or almost all permanent teeth, respectively. Abnormal nail phenotype was reported in two individuals: Individual IV who had absent nails at birth and individual VI who had mild hyponychia and brittle nails. None of the patients could be classified as a specific ED entity [22].

Unexpectedly, 6 patients shared a facial feature of a thin upper lip seen in all but individual Ib (Fig. 1.). Furthermore, individual IV had a dished-in facial profile as reported previously in association with WNT10Aassociated tooth agenesis [14]. No patient reported any systemic conditions.

\section{Genotyping}

Genetic analysis of exons and their flanking sequences of WNT10A was performed as described previously [11], and the results revealed bi-allelic mutations in all seven individuals. All parents were found to be heterozygous. Altogether, three different mutated

Table 1 Clinical data in seven individuals with bi-allelic WNT10A mutations

\begin{tabular}{|c|c|c|c|c|c|c|c|c|}
\hline Patients & la & $\mathrm{lb}$ & $\|$ & III & IV & V & VI & Total \\
\hline Age at radiographic examination & 8.5 & 7.5 & 8.0 & 6.0 & 16.5 & 6.5 & 7.0 & \\
\hline Tooth agenesis in primary dentition & 3 & 0 & 2 & 2 & 0 & 0 & 0 & 7 \\
\hline Tooth agenesis in permanent dentition & 28 & 23 & 26 & 16 & 15 & 19 & 22 & 149 \\
\hline Tapered maxillary central incisors & - & 2 & 2 & 2 & 1 & 0 & 2 & 9 \\
\hline \multirow[t]{2}{*}{ Molars available for analysis of taurodontism ${ }^{a}$} & 75 & 75 & 75 & - & 36 & 75 & 75 & \\
\hline & 85 & 85 & 85 & 85 & 46 & 85 & 85 & 13 \\
\hline Molars with taurodontism ${ }^{a}$ & 1 & 2 & 2 & 1 & 2 & 1 & 2 & 11 \\
\hline Hyperhidrosis of palms and soles & + & + & - & + & - & + & + & 5 \\
\hline Coarse hair structure and/or light/scarce/brittle hair & + & + & + & + & + & + & + & 7 \\
\hline Nail abnormalities & - & - & - & - & + & - & + & 2 \\
\hline Dry skin & + & + & + & + & - & + & + & 6 \\
\hline No. of affected ectodermal appendages & 3 & 3 & 2 & 3 & 2 & 3 & 4 & mean 2.9 \\
\hline
\end{tabular}

${ }^{\mathrm{a}}$ All were primary molars except for individual IV who had resorbed primary second molars and erupted permanent first molars 
alleles were identified resulting in p.C107X, p.F228I and p.E222X (Fig. 1). These three WNT10A variants have been reported earlier $[4,9]$.

\section{Discussion}

We investigated seven individuals with severe oligodontia for their primary dentition, presence of ectodermal symptoms and WNT10A mutations. The selection of patients was based on severe oligodontia of permanent dentition and suspected but yet unspecified ED. Following assessment of primary dentition and presence of other ectodermal symptoms, the patients were analyzed for WNT10A gene variants. $\mathrm{Bi}$-allelic mutations were confirmed in all seven individuals. In total, three different WNT10A alleles were detected that predict the previously described variants C107X, E222X and F228I. Clinical investigations at age 6-14 years revealed that the most distinctive initial sign in all examined individuals was the presence of numerous primary teeth in areas without succedaneous teeth. In total, seven primary incisors were missing from agenesis in three out of seven individuals, $5 \%$ of all primary teeth, compared to $76 \%$ of permanent teeth (mean 21.3). Notably, all three females but none of the males had primary tooth agenesis. This observed gender difference for absent primary teeth is significant $(p=0.02)$ but needs to be confirmed in larger and randomized cohorts. The most stable teeth were the maxillary central incisors, which were missing in only one individual. Severe dental abnormalities were found in individual Ia, who was missing three primary teeth and all permanent teeth, as well as individual II, missing two primary teeth and 26 permanent teeth, all but the maxillary central incisors.

Notably, all well-preserved primary incisors examined were peg teeth. Similarly, the permanent maxillary central incisors were severely tapered in $75 \%$ that were present. Taurodontism of primary or permanent molars was found in all of the examined individuals using biometric analysis from panoramic radiographs. This method is superior and more sensitive than cursory examination, especially in cases of milder forms of taurodontism [23]. The most evident signs were found in the single individual in whom permanent molars were assessed. In mandibular molars from either the primary or permanent dentitions present in each individual $84.6 \%$ were taurodontic. However, taurodontism is frequently found in individuals with hypodontia [21], with or without ED.

Besides tooth agenesis and misshaped teeth, all patients presented with an overlapping spectrum of mostly mild or previously unnoticed ectodermal symptoms including hyperhidrosis of palms and soles, aberrant hair texture, dry skin and nail dystrophy. None of the patients presented with the typical phenotype of $E D A$ or EDAR-induced hypohidrotic ED [24, 25] and the ectodermal signs and symptoms were incongruous with any defined ED-entity. The spectrum of ectodermal symptoms among our seven patients is consistent with that previously reported in patients with bi-allelic WNT10A mutations $[3-6,8,10]$, and even in some patients with mono-allelic mutations $[4,6,8,11,12]$. Notably, with the exception of tooth agenesis, the signs and symptoms from ectodermal appendages in a majority of individuals in our cohort were subtle and in several cases even not experienced as abnormal by the study persons or their parents. Furthermore, we confirmed that carriers for bi-allelic WNT10A mutations have more primary teeth in positions where permanent teeth are missing [4]. Thus, in children with severe tooth agenesis it is important to consider mild or neglected symptoms from other ectodermal appendages. The molecular basis for the broad range of clinical expression due to WNT10A deficiency, from completely asymptomatic carriers to severely disabling ED entities, is yet unclear [10]. Interestingly, a thin upper lip was observed in all but one individual in our study. We observed a similar feature in photographs in three out of five patients from the study by Bohring et al., 2009 [4] Supplemental data]. This sign is distinct from the typical facial phenotype of $E D A$ - and EDAR-induced ED and further studies are now needed to confirm this observation in carriers of WNT10A mutations.

Besides the critical role in the development of ectodermal lineages, increased expression of WNT10A has previously been implicated in a variety of cancers by up-regulation of the $\beta$-catenin pathway $[1,26]$. In contrast, the structural WNT10A mutations known to be associated with abnormal development of ectodermal tissues are predicted to disrupt or reduce WNT mediated signaling [27]. Taken together, this is consistent with the fact that patients with structural WNT10A mutations are not reported to have increased risk for cancer.

Our study shows that bi-allelic WNT10A mutations are associated with a full or nearly full set of primary teeth with typical peg-shaped crowns of the mandibular incisors, persisting primary teeth in areas with missing permanent successors, and extensive tooth agenesis (oligodontia) in the permanent dentition, often with severely tapered maxillary central incisors. This is consistent with and adds to the dental features described by Bohring et al., 2009 [4], Supplemental data]. Furthermore, in combination with mild abnormalities in other ectodermal appendages, this typical dental pattern strongly suggests a WNT10A-associated ED that differs from what is seen in $E D A$ - and $E D A R$-induced hypohidrotic ED. 


\section{Conclusions}

We conclude that overt abnormalities in both primary and permanent dentitions together with other ectodermal symptoms can serve as predictors for WNT10A deficiency. Thus, individuals with bi-allelic WNT10A mutations may be recognized early in life by combining information of both dentitions as well as other ectodermal features of importance for diagnosis, prognosis and counselling.

\section{Abbreviations}

CB:R: Crown-body:root length; ED: Ectodermal dysplasia; PCR: Polymerase chain reaction; UCSC: University of California Santa Cruz; UTR: Untranslated region

\section{Acknowledgements}

We thank the patients and their families for participating in this research. We are also indebted to Marie Danell and Eine Ståhl, specialists in dentomaxillofacial radiology, The Institute for Postgraduate Dental Education, Jönköping, Sweden, for valuable assistance in assessment of taurodontism.

\section{Funding}

This work was supported by grants from the Swedish Research Council (grant 2015-02424 to N.D.), the Thuréus foundation for oral and dental research and Science for Life Laboratory, Uppsala University.

\section{Availability of data and materials}

All data supporting our findings are included in the manuscript.

\section{Authors' contributions}

$\mathrm{BB}$ and JN performed the clinical data collection and assessment of taurodontismXZ performed the genetic analyses. All authors participated in writing, design and interpretation of this report. BB wrote the initial draft of the manuscript which was edited for important intellectual content by JK and ND. All authors have given final approval of this version and all authors are accountable for the accuracy and integrity of this work.

\section{Competing interests}

The authors declare that they have no competing interests.

\section{Consent for publication}

Oral and written consent for publication has been obtained from all study subjects or their parents

\section{Ethics approval and consent to participate}

The study was carried out under ethical approval from the Ethics committee, Linköping University, Sweden, approval number Dnr 02-312. Oral informed consent to participate has been obtained from all study subjects or their parents.

\section{Author details}

${ }^{1}$ National Oral Disability Centre for Rare Disorders, The Institute for Postgraduate Dental Education, P.O. Box 1030SE- 55111 Jönköping, Sweden. ${ }^{2}$ School of Health and Welfare, Jönköping University, Jönköping, Sweden. ${ }^{3}$ Department of Immunology, Genetics and Pathology, Uppsala University, Uppsala, Sweden. ${ }^{4}$ Science for Life Laboratory, Uppsala University, Uppsala, Sweden.

Received: 29 April 2016 Accepted: 15 November 2016 Published online: 24 November 2016

\section{References}

1. Clevers H, Nusse R. Wnt/beta-catenin signaling and disease. Cell. 2012;149: 1192-205.

2. Jernvall J, Thesleff I. Tooth shape formation and tooth renewal: evolving with the same signals. Development. 2012;139:3487-97.

3. Adaimy L, Chouery E, Megarbane H, Mroueh S, Delague V, Nicolas E, Belguith $H$, de Mazancourt P, Megarbane A. Mutation in WNT10A is associated with an autosomal recessive ectodermal dysplasia: the odontoonycho-dermal dysplasia. Am J Hum Genet. 2007;81:821-8.

4. Bohring A, Stamm T, Spaich C, Haase C, Spree K, Hehr U, Hoffmann M, Ledig S, Sel S, Wieacker P, et al. WNT10A mutations are a frequent cause of a broad spectrum of ectodermal dysplasias with sex-biased manifestation pattern in heterozygotes. Am J Hum Genet. 2009:85:97-105

5. Nawaz S, Klar J, Wajid M, Aslam M, Tariq M, Schuster J, Baig SM, Dahl N. WNT10A missense mutation associated with a complete odonto-onychodermal dysplasia syndrome. Eur J Hum Genet. 2009;17:1600-5.

6. Cluzeau C, Hadj-Rabia S, Jambou M, Mansour S, Guigue P, Masmoudi S, Bal E, Chassaing N, Vincent MC, Viot G, et al. Only four genes (EDA1, EDAR, EDARADD, and WNT10A) account for $90 \%$ of hypohidrotic/anhidrotic ectodermal dysplasia cases. Hum Mutat. 2011;32:70-2.

7. Kantaputra P, Kaewgahya M, Jotikasthira D, Kantaputra W. Tricho-odontoonycho-dermal dysplasia and WNT10A mutations. Am J Med Genet A. 2014;164A:1041-8

8. Van den Boogaard MJ, Creton M, Bronkhorst $Y$, Van der Hout A, Hennekam E, Lindhout D, Cune M, van Amstel HK P. Mutations in WNT10A are present in more than half of isolated hypodontia cases. J Med Genet. 2012:49:327-31.

9. Arte S, Parmanen S, Pirinen S, Alaluusua S, Nieminen P. Candidate gene analysis of tooth agenesis identifies novel mutations in six genes and suggests significant role for WNT and EDA signaling and allele combinations. PLoS One. 2013:8:e73705.

10. Plaisancie J, Bailleul-Forestier I, Gaston V, Vaysse F, Lacombe D, HolderEspinasse M, Abramowicz M, Coubes C, Plessis G, Faivre L, et al. Mutations in WNT10A are frequently involved in oligodontia associated with minor signs of ectodermal dysplasia. Am J Med Genet A. 2013;161:671-8.

11. Arzoo PS, Klar J, Bergendal B, Norderyd J, Dahl N. WNT10A mutations account for (1/4) of population-based isolated oligodontia and show phenotypic correlations. Am J Med Genet A. 2014;164A:353-9.

12. Mues G, Bonds J, Xiang L, Vieira AR, Seymen F, Klein O, D'Souza RN. The WNT10A gene in ectodermal dysplasias and selective tooth agenesis. Am J Med Genet A. 2014;164A:2455-60.

13. Tziotzios C, Petrof G, Liu L, Verma A, Wedgeworth EK, Mellerio JE, McGrath JA. Clinical features and WNT10A mutations in seven unrelated cases of Schopf-Schulz-Passarge syndrome. Br J Dermatol. 2014;171:1211-4.

14. Vink CP, Ockeloen CW. ten Kate S, Koolen DA, Ploos van Amstel JK, KuijpersJagtman AM, van Heumen CC, Kleefstra T, Carels CE. Variability in dentofacial phenotypes in four families with WNT10A mutations. Eur J Hum Genet. 2014;22:1063-70.

15. Massink MP, Creton MA, Spanevello F, Fennis WM, Cune MS, Savelberg SM, Nijman IJ, Maurice MM, van den Boogaard MJ, van Haaften G. Loss-ofFunction Mutations in the WNT Co-receptor LRP6 Cause AutosomalDominant Oligodontia. Am J Hum Genet. 2015;97:621-6.

16. Kimura R, Watanabe C, Kawaguchi A, Kim YI, Park SB, Maki K, Ishida H, Yamaguchi T. Common polymorphisms in WNT10A affect tooth morphology as well as hair shape. Hum Mol Genet. 2015;24:2673-80.

17. Yang J, Wang SK, Choi M, Reid BM, Hu Y, Lee YL, Herzog CR, Kim-Berman H, Lee M, Benke PJ, et al. Taurodontism, variations in tooth number, and misshapened crowns in Wnt10a null mice and human kindreds. Mol Genet Genomic Med. 2015;3:40-58

18. Terezhalmy GT, Riley CK, Moore WS. Clinical images in oral medicine and maxillofacial radiology. Taurodontism. Quintessence Int. 2001;32:254-5.

19. Lexner MO, Bardow A, Hertz JM, Nielsen LA, Kreiborg S. Anomalies of tooth formation in hypohidrotic ectodermal dysplasia. Int J Paediatr Dent. 2007;17:10-8.

20. Seow WK, Lai PY. Association of taurodontism with hypodontia: a controlled study. Pediatr Dent. 1989:11:214-9.

21. Kan WY, Seow WK, Holcombe T. Taurodontism in children with hypodontia and supernumerary teeth: a case control study. Pediatr Dent. 2010;32:134-40.

22. Visinoni AF, Lisboa-Costa T, Pagnan NA, Chautard-Freire-Maia EA Ectodermal dysplasias: clinical and molecular review. Am J Med Genet A. 2009:149A:1980-2002.

23. Hegde V, Anegundi RT, Pravinchandra KR. Biometric Analysis - A Reliable Indicator for Diagnosing Taurodontism using Panoramic Radiographs. J Clin Diagn Res. 2013;7:1779-81. 
24. Bergendal B. Orodental manifestations in ectodermal dysplasia-a review, Am J Med Genet A. 2014;164A:2465-71.

25. Kieri CF, Bergendal B, Lind LK, Schmitt-Egenolf M, Stecksen-Blicks C. EDARinduced hypohidrotic ectodermal dysplasia: a clinical study on signs and symptoms in individuals with a heterozygous C.1072C > T mutation. BMC Med Genet. 2014;15:57.

26. Long A, Giroux V, Whelan KA, Hamilton KE, Tetreault MP, Tanaka K, Lee JS, Klein-Szanto AJ, Nakagawa H, Rustgi AK. WNT10A promotes an invasive and self-renewing phenotype in esophageal squamous cell carcinoma. Carcinogenesis. 2015;36:598-606.

27. Liu F, Millar SE. Wnt/beta-catenin signaling in oral tissue development and disease. J Dent Res. 2010;89:318-30.

Submit your next manuscript to BioMed Central and we will help you at every step:

- We accept pre-submission inquiries

- Our selector tool helps you to find the most relevant journal

- We provide round the clock customer support

- Convenient online submission

- Thorough peer review

- Inclusion in PubMed and all major indexing services

- Maximum visibility for your research

Submit your manuscript at www.biomedcentral.com/submit
Biomed Central 